\title{
Recurrent pneumonia due to a foreign body in the lower respiratory tract
}

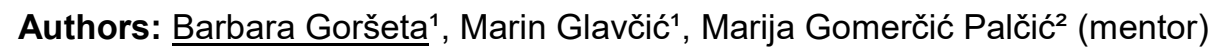

\author{
${ }^{1}$ School of Medicine, University of Zagreb, Zagreb, Croatia \\ ${ }^{2}$ Department for clinical immunology, pulmonology and rheumatology, University Hospital Center \\ Sestre Milosrdnice, Zagreb, Croatia
}

DOI: https://doi.org/10.26800/LV-142-supp5-2

\section{Background:}

Seventy-year-old male was admitted to University Hospital Center Sestre milosrdnice, Department for clinical immunology, pulmonology and rheumatology for further assessment after nearly eight months of recurrent migratory pneumonias impacting both lower lobes of the lung.

\section{Case presentation:}

Six years ago patient had a stroke that resulted with left hemiparesis that never resolved. During the eight month period prior hospitalization he was treated with four different lines of antibiotics in seven occasions (some were prescribed even few different times). During that time he was examined by six different physicians, pulmonologists and primary healthcare specialist, and had repeated chest-X-ray (CXR) exams ten times and in the end multi-slice computed tomography (MSCT) of the thorax was done. Also, he was tested negative for SARS-CoV-2 twice. Patient's condition would improve during antibiotic treatment, but soon after finishing the therapy his condition worsened. At the admission he had strong cough with yellow colored sputum and complained of dyspnea at rest as well as in exertion. He wasn't febrile possibly due to levofloxacin he had been using twenty days prior admission. Diffuse sharpened breathing sound and prolonged expiration could be heard on auscultation with expiratory wheezing on the right lung. CXR and MSCT showed multiple inflammatory consolidations of pulmonary parenchyma in both left and right lung and right pleural effusion was detected. Lung function test implicated possible restrictive pattern (FVC 62\%) and moderate degree of obstruction (FEV1 53\%, FEV1/FVC 0.65). Fiberbronchoscopy was performed and four cherry pits were identified and extracted. Following foreign body removal patient had normal physical examination of the lungs as well as lung function tests.

\section{Conclusion:}

This case shows that patient with inadequate reflexes and repeated pneumonias in relatively short period should undergo bronchoscopy with suspicion of the foreign body aspiration and could avoid unnecessary antibiotic treatment and unwarranted diagnostic procedures (e.g. CXR).

Keywords: foreign body, pneumonia, stroke 